\title{
Extending and Merging the Purple Crow Lidar Temperature Climatologies Using the Inversion Method
}

\author{
Ali Jalali ${ }^{1}$, R.J.Sica ${ }^{1,2,3}$, P. S. Argall ${ }^{1,4}$ \\ ${ }^{1}$ Department of Physics and Astronomy, University of Western Ontario, London, Canada \\ *Email:ajalali3@uwo.ca \\ ${ }^{2}$ Visiting Scientist, MétéoSuisse, Payerne, Switzierland \\ ${ }^{3}$ Visiting Scientist, Environmental Fluid Dynamics Laboratory, École Polytechnique Fédérale de \\ Lausanne, Switzerland \\ ${ }^{4}$ School of Applied Science and Technology, Fanshawe College, London, Ontario
}

\begin{abstract}
Rayleigh and Raman scatter measurements from The University of Western Ontario Purple Crow Lidar (PCL) have been used to develop temperature climatologies for the stratosphere, mesosphere, and thermosphere using data from 1994 to 2013 (Rayleigh system) and from 1999 to 2013 (vibrational Raman system). Temperature retrievals from Rayleigh-scattering lidar measurements have been performed using the methods by Hauchecorne and Chanin (1980; henceforth HC) and Khanna et al. (2012). Argall and Sica (2007) used the HC method to compute a climatology of the PCL measurements from 1994 to 2004 for 35 to $110 \mathrm{~km}$, while Iserhienrhien et al. (2013) applied the same technique from 1999 to 2007 for 10 to $35 \mathrm{~km}$. Khanna et al. (2012) used the inversion technique to retrieve atmospheric temperature profiles and found that it had advantages over the HC method. This paper presents an extension of the PCL climatologies created by Argall and Sica (2007) and Iserhienrhien et al. (2013). Both the inversion and $\mathrm{HC}$ methods were used to form the Rayleigh climatology, while only the latter was adopted for the Raman climatology. Then, two different approaches were used to merge the climatologies from 10 to $110 \mathrm{~km}$. Among four different functional identities, a trigonometric hyperbolic relation results in the best choice for merging temperature profiles between the Raman and Low level Rayleigh channels, with an estimated uncertainty of $0.9 \mathrm{~K}$ for merging temperatures. Also, error function produces best result with uncertainty of $0.7 \mathrm{~K}$ between the Low Level Rayleigh and High Level Rayleigh channels. The
\end{abstract}

results show that the temperature climatologies produced by the $\mathrm{HC}$ method when using a seed pressure are comparable to the climatologies produced by the inversion method. The Rayleigh extended climatology is slightly warmer below 80 $\mathrm{km}$ and slightly colder above $80 \mathrm{~km}$. There are no significant differences in temperature between the extended and the previous Raman channel climatologies. Through out this study, we discuss the data collected by PCL for a long term (19942013) up to $110 \mathrm{~km}$. Moreover, different merging functions in various methods were used to merge the temperature climatologies for different channels.

\section{INTRODUCTION}

Rayleigh and Raman lidar systems are designed to detect photon backscatter from the atmosphere. These systems provide high temporal and vertical resolutions, making them suitable candidates to study the climatology of the atmosphere. The Purple Crow Lidar (PCL)(Sica et al. [1995, 2001]) is currently located at the Environmental Science Western Field Station (43.07 N, 81.33W, 275 m) near The University of Western Ontario in London, Canada and has been operating since 1992. Now, a Nd:YAG solid state laser is used to produce $532 \mathrm{~nm}$ beam with a repetition rate of 30 $\mathrm{Hz}$ with $1000 \mathrm{~mJ}$ energy per pulse for Rayleigh and Raman lidars. The conventional method for retrieving temperature from lidar measurements was first proposed by Hauchecorne and Chanin in 1980. In previous PCL climatologies, this method 
was used for obtaining temperature from the PCL measurements. One important difference in this study is a grid search optimization (inversion) technique, which was used to analyze Rayleigh channel data in order to improve the deficiencies of the HC method. The PCL is comprised of three channels: Raman, analog Rayleigh and digital Rayleigh. The Raman channel measures the temperature of the upper troposphere and lower stratosphere with data collected from 1999 to the present from 10 to $45 \mathrm{~km}$. The digital Rayleigh and analog Rayleigh channels measure temperature in the upper mesosphere and lower thermosphere, which the measurements have been conducted since 1994. Currently, the PCL has two PMTs for the Rayleigh channel. The new one, labeled as Rayleigh Licel (High level Rayleigh), and old Rayleigh channel labeled as the Rayleigh Hamamatsu (Low level Rayleigh). The old Rayleigh channel was used for calibration and alignment while testing the linearity of the new Licel system. These three channels were used to perform PCL climatology.

\section{METHODOLOGY}

Different techniques have been suggested to retrieve temperature from lidar measurements. There are two methods for retrieving temperature from lidar measurements, these methods are called the conventional method which was first proposed by Hauchecorne and Chanin in 1980 and the inversion method presented by Khanna et al., 2012. Each approach has its own deficiencies and benefits. In the $\mathrm{HC}$ method, using hydrostatic equilibrium, the ideal gas law and the lidar equation, we can find a relation between observed lidar output and the temperature at each altitude in the lidar range. The details of this technique are described in Hauchecorne and Chanin (1980). In order to integrate the pressure relation from the top to bottom, it is necessary to know the value of the seed pressure or seed temperature at the top. Because the temperature and density measurements in the thermosphere have large fluctuations, the initial guess of the pressure or temperature at the top has a large uncertainty, and this uncertainty will be carried through the computation process of retrieved temperatures in lower altitudes. The starting point at the top for the Rayleigh channel is set between 100 to 110 $\mathrm{km}$, and the corresponding pressure is obtained from the CIRA model. Because of the uncertainty in the value of the seed pressure or temperature, the uncertainty of the retrieved temperatures is large for the first $15 \mathrm{~km}$ below the highest altitude. Therefore, it is common to remove the first 10 to $15 \mathrm{~km}$ from the temperature profiles in order to achieve more accurate results. However, we lose valuable information in the top layers-the disadvantage of this method. One solution for this deficiency of the conventional method is the inversion method. The inversion method is an optimization scheme that uses an iterative approach to estimate the true value of parameters by starting from an initial guessed value of the parameter vector and proceeding by updating the parameters with their improved values step by step until it reaches the best estimate for temperature. In the inversion method, still we use the seed pressure but contrary to the conventional method, the seed pressure is at the bottom and the integration is upward. In the upward integration method, the seed pressure and temperature, which are defined to be between 30 to $35 \mathrm{~km}$, can be measured accurately using a variety of methods. Therefore, using this method will decrease the uncertainties. Since the inversion method does not provide statistical model or model parameter uncertainties, the uncertainties of its results can be estimated using Monte Carlo techniques, whereas in the conventional method the uncertainties are calculated analytically. Details of the inversion method are explained in Khanna et al., (2012). In order to extend our results to include an extra 10 $\mathrm{km}$ on top, the inversion method is used to perform temperature climatology for the PCL High Level Rayleigh channel. In this study, the Argall and Sica methodology was followed to form the temperature climatologies where the methodology is explained in detail in Argall and Sica (2007). The temperature climatologies were calculated for separately for the High Level Rayleigh, Low Level Rayleigh and Raman channels. 


\section{RESULTS}

The temperature climatologies were performed for High Level Rayleigh, Low Level Rayleigh and Raman channel. The PCL channels overlap in some regions in altitude. In overlapping regions, the temperature should be identical but there are differences between them. Therefore, the different ranges are combined to have an individual profile for the entire atmosphere from $10 \mathrm{~km}$ up to 110 $\mathrm{km}$ and are known as "merged" profile. Retrieved temperatures for each channel have more statistical accuracy at the bottom rather than the top of each profile. As a result, some regions for each channel are more reliable in a common area. Therefore, instead of using simple averages between temperatures, four functions were chosen to merge the temperature profiles between different channels: sine/cosine, simple linear, error function (erf), and hyperbolic functions. PCL measurements have been obtained with better or worse precision because each lidar channel measurement in a common area has different precision. In this case, the data points are expressed by assuming the same distribution with the same mean but with different standard deviations. Therefore the weighted average of the measurements is the most probable value. The weighted averages for the Raman and LLR channel between 30 to $35 \mathrm{~km}$, as well as LLR and HLR channel between 35 to $40 \mathrm{~km}$, were calculated. The weighted average result was chosen as a reference for evaluating merging functions. The related errors for the PCL are a combination of statistical errors due to the photon counting and systematic errors due to applied corrections. Although, the applied corrections in merging area are fairly small. Consequently, the weighted average function was chosen as a reference for the evaluation of the merging functions. Two different approaches were used to evaluate the merging functions and are explained in following sections. In the first approach, in order to extend the range of lidar measurements, temperature climatologies from different channels were merged together. First, merging altitudes were found by considering several factors, including: photon count linearity, temperature standard deviation, and temperature differences between different channels. The best altitude range between the Raman and LLR channels was
30 to $35 \mathrm{~km}$ and between the LLR and HLR was 35 to $40 \mathrm{~km}$. Then temperature climatologies were merged together using each merging function between the Raman and LLR channels, as well as LLR and HLR channels. The differences between weighted average temperatures and merged parts are considered as matrices, where these matrices represent the average uncertainty for each function for an entire year. In order to evaluate each function, the standard deviations of these matrices were calculated. The results show that the hyperbolic function is the best function for merging the Raman and LLR channel with uncertainty of $0.9 \mathrm{~K}$ and the error function is a better function for the LLR and HLR with an uncertainty of $0.7 \mathrm{~K}$. After finding the best merging functions between the different channels, the combined PCL climatology was found.

The second approach involved merging the nightly temperature profiles together first and then creating the temperature climatology. In the first step the common dates among the Raman, LLR and HLR channels were found a total of 297 nights. The second method was unlike the last approach, where the merged area was constant. In the second approach, the critical height was calculated for each night, and then the merged area was defined as the critical height plus 5 $\mathrm{km}$, if this summation was less than $50 \mathrm{~km}$ for merging HLR-LLR. In the case that the result of the critical height $+5 \mathrm{~km}$ was larger than $50 \mathrm{~km}$ for the HLR-LLR merging, the merged area was set between 45 to $50 \mathrm{~km}$. The LLR-Raman merged area was chosen between 30 and $35 \mathrm{~km}$. Temperature climatology differences between the weighted average result and each function was calculated. Finally, the standard deviation of each difference matrix was determined to be the uncertainty for each function. The results for this approach are consistent with the first approach. Figure 1 shows the PCL merged temperature climatology. The white area in this figure is related to the lack of data.

Finally, the new extended climatology was compared to previous climatology. The results revel the increase in temperature between 35 to 80 $\mathrm{km}$ and decrease above $80 \mathrm{~km}$. Also there are no significant differences in temperature between the 
extended and the previous Raman channel climatologies.

\section{CONCLUSIONS}

The inversion method method showed a significant improvement in the integration of temperature profiles using the lidar equation when compared to the conventional technique. In this study, temperature climatologies were calculated for all available PCL Rayleigh measurements in order to investigate and evaluate the new method, as well as update the PCL temperature climatology using inversion method. The comparison between the conventional and inversion method results revealed that given the same uncertainty level, the inversion method had the advantage of gaining approximately an about extra $10 \mathrm{~km}$ in retrieved temperatures. Between the tested functions for merging the different temperature channels, the hyperbolic function had the best result for merging the Raman and LLR channels, with an uncertainty of $0.9 \mathrm{~K}$. The error function yielded a better result for merging temperatures between the LLR and HLR channels, with a $0.7 \mathrm{~K}$ uncertainty. The different approaches for evaluating merging functions confirmed their results.

\section{ACKNOWLEDGEMENT}

Ali Jalali would like to thank the NSERC CREATE Training Program in Arctic Atmospheric Science and the Canadian Network for the Detection of Atmospheric Change (CANDAC) for their support. We would also like to acknowledge the support of the Canadian National Science and Engineering Research Council.

\section{REFERENCES}

[1] Argall, P. S. and R. J. Sica. A comparison of rayleigh and sodium lidar temperature climatologies. Ann. Geophys., 25:27-35, 2007.

[2] Hauchecorne, A., M. Chanin, and P. Keckhut.
Climatology and trends of the middle atmospheric temperature $(33-87 \mathrm{~km})$ as seen by rayleigh lidar over the south of france. J. Geophys. Res., 96:5169-5183, 1991.

[3] Iserhienrhien, B., R. J. Sica, and P. S. Argall. A 7-year lidar temperature climatology of the mid-latitude upper troposphere and stratosphere. Atmosphere-Ocean, 51:532-540, 2013.

[4] Khanna, Jaya, Justin Bandoro, R. J. Sica, and C. Thomas McElroy. New technique for retrieval of atmospheric temperature profiles from rayleigh-scatter lidar measurements using nonlinear inversion. Applied Optics, 51:7945-52, 2012.

[4] Sica, R. J., S. Sargoytchev, P. S. Argall, E. F. Borra, L. Girard, C. T. Sparrow, and S. Flatt. Lidar measurements taken with a large aperture liquid mirror. 1. rayleigh scatter system. Applied Optics, 34:6925-6936, 1995.

[5] Sica, R. J., Z. A. Zylawy, and P.S. Argall. Ozone corrections for rayleigh-scatter temperature determinations in the middle atmosphere. Journal of Atmospheric and Oceanic Technology, 18:1223-1228, 2001.

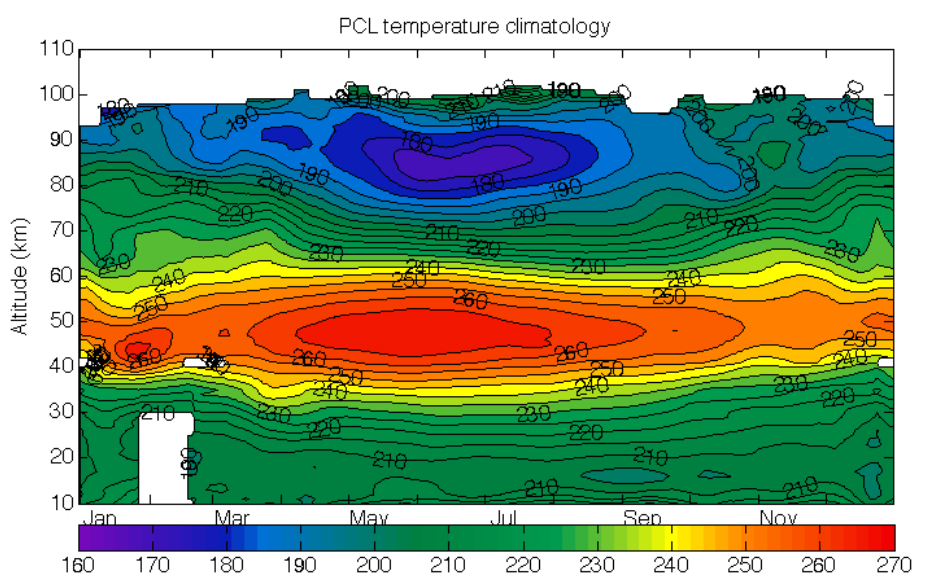

Figure 1- The PCL temperature climatology. 